УДК 517.53

\author{
S. G. KIM
}

\title{
THE NORMING SET OF A SYMMETRIC BILINEAR FORM ON THE PLANE WITH THE SUPREMUM NORM
}

S. G. Kim. The norming set of a symmetric bilinear form on the plane with the supremum norm, Mat. Stud. 55 (2021), 171-180.

An element $\left(x_{1}, \ldots, x_{n}\right) \in E^{n}$ is called a norming point of $T \in \mathcal{L}_{s}\left({ }^{n} E\right)$ if $\left\|x_{1}\right\|=\cdots=$ $\left\|x_{n}\right\|=1$ and $\left|T\left(x_{1}, \ldots, x_{n}\right)\right|=\|T\|$, where $\mathcal{L}_{s}\left({ }^{n} E\right)$ denotes the space of all symmetric continuous $n$-linear forms on $E$. For $T \in \mathcal{L}_{s}\left({ }^{n} E\right)$, we define

$$
\operatorname{Norm}(T)=\left\{\left(x_{1}, \ldots, x_{n}\right) \in E^{n}:\left(x_{1}, \ldots, x_{n}\right) \text { is a norming point of } T\right\} .
$$

$\operatorname{Norm}(T)$ is called the norming set of $T$. We classify $\operatorname{Norm}(T)$ for every $T \in \mathcal{L}_{s}\left({ }^{2} l_{\infty}^{2}\right)$.

1. Introduction. Let $n \in \mathbb{N}, n \geq 2$. We write $S_{E}$ for the unit sphere of a Banach space $E$. We denote by $\mathcal{L}\left({ }^{n} E\right)$ the Banach space of all continuous $n$-linear forms on $E$ endowed with the norm $\|T\|=\sup _{\left(x_{1}, \cdots, x_{n}\right) \in S_{E} \times \cdots \times S_{E}}\left|T\left(x_{1}, \cdots, x_{n}\right)\right| \cdot \mathcal{L}_{s}\left({ }^{n} E\right)$ denotes the closed subspace of all continuous symmetric $n$-linear forms on $E$. An element $\left(x_{1}, \ldots, x_{n}\right) \in E^{n}$ is called a norming point of $T$ if $\left\|x_{1}\right\|=\cdots=\left\|x_{n}\right\|=1$ and $\left|T\left(x_{1}, \ldots, x_{n}\right)\right|=\|T\|$.

For $T \in \mathcal{L}\left({ }^{n} E\right)$, we define

$$
\operatorname{Norm}(T)=\left\{\left(x_{1}, \ldots, x_{n}\right) \in E^{n}:\left(x_{1}, \ldots, x_{n}\right) \text { is a norming point of } T\right\} .
$$

$\operatorname{Norm}(T)$ is called the norming set of $T$. Notice that $\left(x_{1}, \ldots, x_{n}\right) \in \operatorname{Norm}(T)$ if and only if $\left(\epsilon_{1} x_{1}, \ldots, \epsilon_{n} x_{n}\right) \in \operatorname{Norm}(T)$ for some $\epsilon_{k}= \pm 1(k=1, \ldots, n)$. Indeed, if $\left(x_{1}, \ldots, x_{n}\right) \in$ $\operatorname{Norm}(T)$, then

$$
\left|T\left(\epsilon_{1} x_{1}, \ldots, \epsilon_{n} x_{n}\right)\right|=\left|\epsilon_{1} \cdots \epsilon_{n} T\left(x_{1}, \ldots, x_{n}\right)\right|=\left|T\left(x_{1}, \ldots, x_{n}\right)\right|=\|T\|,
$$

which shows that $\left(\epsilon_{1} x_{1}, \ldots, \epsilon_{n} x_{n}\right) \in \operatorname{Norm}(T)$. If $\left(\epsilon_{1} x_{1}, \ldots, \epsilon_{n} x_{n}\right) \in \operatorname{Norm}(T)$ for some $\epsilon_{k}=$ $\pm 1(k=1, \ldots, n)$, then

$$
\left(x_{1}, \ldots, x_{n}\right)=\left(\epsilon_{1}\left(\epsilon_{1} x_{1}\right), \ldots, \epsilon_{n}\left(\epsilon_{n} x_{n}\right)\right) \in \operatorname{Norm}(T) .
$$

The following examples show that $\operatorname{Norm}(T)$ is either equal to the empty set or it is an infinite set.

Examples. (a) Let

$$
T\left(\left(x_{i}\right)_{i \in \mathbb{N}},\left(y_{i}\right)_{i \in \mathbb{N}}\right)=\sum_{i, j \in \mathbb{N}} a_{i j} x_{i} y_{j} \in \mathcal{L}_{s}\left({ }^{2} c_{0}\right)
$$

2010 Mathematics Subject Classification: 46A22.

Keywords: norming points; symmetric bilinear forms.

doi:10.30970/ms.55.2.171-180

(C) S. G. Kim, 2021 
for some $a_{i j} \in \mathbb{R}$ such that $\|T\|=\sum_{i, j \in \mathbb{N}}\left|a_{i j}\right|$. We claim that if $A=\left\{(i, j) \in \mathbb{R}^{2}: a_{i j} \neq 0\right\}$ is an infinite set, then $\operatorname{Norm}(T)=\varnothing$.

Assume that $\operatorname{Norm}(T) \neq \varnothing$. Let $\left(\left(x_{i}\right)_{i \in \mathbb{N}},\left(y_{i}\right)_{i \in \mathbb{N}}\right) \in \operatorname{Norm}(T)$. Then,

$$
\|T\|=\left|T\left(\left(x_{i}\right)_{i \in \mathbb{N}},\left(y_{i}\right)_{i \in \mathbb{N}}\right)\right| \leq \sum_{(i, j) \in A}\left|a_{i j}\right|\left|x_{i} y_{j}\right| \leq \sum_{(i, j) \in A}\left|a_{i j}\right|=\|T\|,
$$

which shows that $\left|x_{i} y_{j}\right|=1$ for all $(i, j) \in A$. Hence, $\left|x_{i}\right|=1$ for infinitely many $i \in \mathbb{N}$ or $\left|y_{j}\right|=1$ for infinitely many $j \in \mathbb{N}$ since $A$ is an infinite set. Therefore, $\left(x_{i}\right)_{i \in \mathbb{N}} \notin c_{0}$ or $\left(y_{i}\right)_{i \in \mathbb{N}} \notin c_{0}$. This is a contradiction. Therefore, $\operatorname{Norm}(T)=\varnothing$.

From this, if

$$
T\left(\left(x_{i}\right)_{i \in \mathbb{N}}, \quad\left(y_{i}\right)_{i \in \mathbb{N}}\right)=\sum_{i=1}^{\infty} \frac{1}{2^{i}} x_{i} y_{i} \in \mathcal{L}_{s}\left({ }^{2} c_{0}\right),
$$

then $\operatorname{Norm}(T)=\varnothing$.

We do not know whether there is some $T \in \mathcal{L}_{s}\left({ }^{2} c_{0}\right)$ such that $\operatorname{Norm}(T)$ is a nonempty finite set.

(b) Let

$$
T\left(\left(x_{i}\right)_{i \in \mathbb{N}}, \quad\left(y_{i}\right)_{i \in \mathbb{N}}\right)=x_{1} y_{1} \in \mathcal{L}_{s}\left({ }^{2} c_{0}\right)
$$

Then,

$\operatorname{Norm}(T)=\left\{\left(\left( \pm 1, x_{2}, x_{3}, \ldots\right),\left( \pm 1, y_{2}, y_{3}, \ldots\right)\right) \in c_{0} \times c_{0}:\left|x_{j}\right| \leq 1,\left|y_{j}\right| \leq 1\right.$ for $\left.j \geq 2\right\}$.

A mapping $P: E \rightarrow \mathbb{R}$ is a continuous $n$-homogeneous polynomial if there exists a continuous $n$-linear form $L$ on the product $E \times \cdots \times E$ such that $P(x)=L(x, \ldots, x)$ for every $x \in E$. We denote by $\mathcal{P}\left({ }^{n} E\right)$ the Banach space of all continuous $n$-homogeneous polynomials from $E$ into $\mathbb{R}$ endowed with the norm $\|P\|=\sup _{\|x\|=1}|P(x)|$.

An element $x \in E$ is called a norming point of $P \in \mathcal{P}\left({ }^{n} E\right)$ if $\|x\|=1$ and $|P(x)|=\|P\|$. For $P \in \mathcal{P}\left({ }^{n} E\right)$, we define

$$
\operatorname{Norm}(P)=\{x \in E: x \text { is a norming point of } P\} .
$$

$\operatorname{Norm}(P)$ is called the norming set of $P$. Notice that $\operatorname{Norm}(P)=\varnothing$ or a finite set or an infinite set.

Recently, Kim [8] classify $\operatorname{Norm}(P)$ for every $P \in \mathcal{P}\left({ }^{2} l_{\infty}^{2}\right)$, where $l_{\infty}^{2}=\mathbb{R}^{2}$ with the supremum norm.

If $\operatorname{Norm}(T) \neq \varnothing, T \in \mathcal{L}\left({ }^{n} E\right)$ is called a norm attaining $n$-linear form and if $\operatorname{Norm}(P) \neq \varnothing$, $P \in \mathcal{P}\left({ }^{n} E\right)$ is called a norm attaining $n$-homogeneoue polynomial (see [3]).

For more details about the theory of multilinear mappings and polynomials on a Banach space, we refer to [5].

Let us introduce a brief history of norm attaining multilinear forms and polynomials on Banach spaces. In 1961 Bishop and Phelps [2] initiated and showed that the set of norm attaining functionals on a Banach space is dense in the dual space. Shortly after, attention was paid to possible extensions of this result to more general settings, specially bounded linear operators between Banach spaces. The problem of denseness of norm attaining functions has moved to other types of mappings like multilinear forms or polynomials. The first result about norm attaining multilinear forms appeared in a joint work of Aron, Finet and Werner [1], where they showed that the Radon-Nikodym property is sufficient for the denseness 
of norm attaining multilinear forms. Choi and Kim [3] showed that the Radon-Nikodym property is also sufficient for the denseness of norm attaining polynomials. Jimenez-Sevilla and Paya [6] studied the denseness of norm attaining multilinear forms and polynomials on preduals of Lorentz sequence spaces.

It seems to be natural and interesting to study about $\operatorname{Norm}(T)$ for $T \in \mathcal{L}_{s}\left({ }^{n} E\right)$. For $m \in$ $\mathbb{N}$, let $l_{\infty}^{m}:=\mathbb{R}^{m}$ with the supremum norm. Notice that for every $T \in \mathcal{L}_{s}\left({ }^{n} l_{\infty}^{m}\right), \operatorname{Norm}(T) \neq \varnothing$ since $S_{l_{\infty}^{m}}$ is compact.

In this paper, we classify $\operatorname{Norm}(T)$ for every $T \in \mathcal{L}_{s}\left({ }^{2} l_{\infty}^{2}\right)$.

\section{Results.}

Theorem A ([7]). Let $T\left(\left(x_{1}, x_{2}\right),\left(y_{1}, y_{2}\right)\right)=a x_{1} y_{1}+b x_{2} y_{2}+c\left(x_{1} y_{2}+x_{2} y_{1}\right) \in \mathcal{L}_{s}\left({ }^{2} l_{\infty}^{2}\right)$. Then,

$$
\|T\|=\max \{|a+b|+2|c|,|a-b|\} .
$$

Notice that if $\|T\|=1$, then $|a| \leq 1,|b| \leq 1,|c| \leq \frac{1}{2}$.

Lemma 1. Let $T\left(\left(x_{1}, x_{2}\right),\left(y_{1}, y_{2}\right)\right)=a x_{1} y_{1}+b x_{2} y_{2}+c\left(x_{1} y_{2}+x_{2} y_{1}\right) \in \mathcal{L}_{s}\left({ }^{2} l_{\infty}^{2}\right)$. Then there exists (unique) $T^{\prime}\left(\left(x_{1}, x_{2}\right),\left(y_{1}, y_{2}\right)\right)=a^{*} x_{1} y_{1}+b^{*} x_{2} y_{2}+c^{*}\left(x_{1} y_{2}+x_{2} y_{1}\right) \in \mathcal{L}_{s}\left({ }^{2} l_{\infty}^{2}\right)$ such that $a^{*}, b^{*}, c^{*} \in\{ \pm a, \pm b, \pm c\}$ with $a^{*} \geq\left|b^{*}\right|$ and $c^{*} \geq 0$ and $\|T\|=\left\|T^{\prime}\right\|$.

Proof. If $a<0$, taking $-T$, we assume that $a \geq 0$. If $|b|>a$, let

$$
T_{1}^{\prime}\left(\left(x_{1}, x_{2}\right),\left(y_{1}, y_{2}\right)\right):=T\left(\left(x_{2}, x_{1}\right),\left(y_{2}, y_{1}\right)\right)=|b| x_{1} y_{1}+a x_{2} y_{2}+c\left(x_{1} y_{2}+x_{2} y_{1}\right) .
$$

Then, $\left\|T_{1}^{\prime}\right\|=\|T\|$. If $c<0$, let

$$
T_{2}^{\prime}\left(\left(\left(x_{1}, x_{2}\right),\left(y_{1}, y_{2}\right)\right):=T_{1}^{\prime}\left(\left(-x_{1}, x_{2}\right),\left(-y_{1}, y_{2}\right)\right)=|b| x_{1} y_{1}+a x_{2} y_{2}+|c|\left(x_{1} y_{2}+x_{2} y_{1}\right) .\right.
$$

Then, $\left\|T_{2}^{\prime}\right\|=\|T\|$. Therefore, we can find a bilinear form $T^{\prime}$ which satisfies the conditions of the theorem.

Lemma 2. Let $T\left(\left(x_{1}, x_{2}\right),\left(y_{1}, y_{2}\right)\right)=a x_{1} y_{1}+b x_{2} y_{2}+c\left(x_{1} y_{2}+x_{2} y_{1}\right) \in \mathcal{L}_{s}\left({ }^{2} l_{\infty}^{2}\right)$. Then, $\left(\left(x_{1}, x_{2}\right), \quad\left(y_{1}, y_{2}\right)\right) \in \operatorname{Norm}(T)$ if and only if $\left(\left(y_{1}, y_{2}\right),\left(x_{1}, x_{2}\right)\right),\left(-\left(x_{1}, x_{2}\right), \quad\left(y_{1}, y_{2}\right)\right)$, $\left(\left(x_{1}, x_{2}\right),-\left(y_{1}, y_{2}\right)\right),\left(-\left(x_{1}, x_{2}\right),-\left(y_{1}, y_{2}\right)\right) \in \operatorname{Norm}(T)$.

Let $T\left(\left(x_{1}, x_{2}\right),\left(y_{1}, y_{2}\right)\right)=a x_{1} y_{1}+b x_{2} y_{2}+c\left(x_{1} y_{2}+x_{2} y_{1}\right) \in \mathcal{L}_{s}\left({ }^{2} l_{\infty}^{2}\right)$. By Lemma 1, without loss of generality we may assume that $a \geq|b|$ and $c \geq 0$.

We are in position to prove the main result of this paper.

Theorem 1. Let $T\left(\left(x_{1}, x_{2}\right),\left(y_{1}, y_{2}\right)\right)=a x_{1} y_{1}+b x_{2} y_{2}+c\left(x_{1} y_{2}+x_{2} y_{1}\right) \in \mathcal{L}_{s}\left({ }^{2} l_{\infty}^{2}\right)$ be such that $\|T\|=1$ with $a \geq|b|$ and $c \geq 0$. Then,

Case 1: $b \geq 0$.

If $c=0=b$, then

$$
\operatorname{Norm}(T)=\{( \pm(1, t), \pm(1, s)):-1 \leq t, s \leq 1\}
$$

If $c=0, b>0$, then

$$
\operatorname{Norm}(T)=\{( \pm(1,1), \pm(1,1)),( \pm(1,-1), \pm(1,-1))\}
$$

If $c>0$, then

$$
\operatorname{Norm}(T)=\{( \pm(1,1), \pm(1,1))\}
$$


Case 2: $b<0$

Subcase 1: $1=|a+b|+2|c|>|a-b|$.

If $c<\frac{1}{2}$, then

$$
\operatorname{Norm}(T)=\{( \pm(1,1), \pm(1,1)\}
$$

If $c=\frac{1}{2}$, then

$$
\operatorname{Norm}(T)=\{( \pm(1,1), \pm(1,1)),( \pm(1,-1), \pm(1,-1))\} .
$$

Subcase 2: $|a+b|+2|c|=|a-b|=1$.

If $a=\frac{1}{2}$, then

$$
\begin{aligned}
\operatorname{Norm}(T)= & \{( \pm(1,-1), \pm(s, 1)),( \pm(s, 1), \pm(1,-1)),( \pm(1, t), \pm(1,1)), \\
& ( \pm(1,1), \pm(1, t)):-1 \leq s, t \leq 1\} .
\end{aligned}
$$

If $\frac{1}{2}<a$, then

$$
\begin{aligned}
\operatorname{Norm}(T)= & \{( \pm(1, t), \pm(1,1)),( \pm(1,1), \pm(1, t)),( \pm(1,1), \pm(1,-1)) \\
& ( \pm(1,-1), \pm(1,1)):-1 \leq t \leq 1\}
\end{aligned}
$$

Subcase 3: $|a+b|+2|c|<|a-b|=1$.

$$
\operatorname{Norm}(T)=\{( \pm(1,-1), \pm(1,1)),( \pm(1,1), \pm(1,-1))\} .
$$

Proof. Let $\left(\left(x_{1}, x_{2}\right),\left(y_{1}, y_{2}\right)\right) \in \operatorname{Norm}(T)$. By Lemma 2, we may assume that $\left(x_{1}=y_{1}=1\right)$ or $\left(x_{1}=y_{2}=1\right)$ or $\left(x_{2}=y_{2}=1\right)$.

Case 1: $b \geq 0$.

By Theorem A,

$$
\|T\|=1=|a+b|+2|c|=a+b+2 c>|a-b|=a-|b| .
$$

Let $c>0$. Suppose that $((1, t),(1, y)) \in \operatorname{Norm}(T)$ for some $t, y \in[-1,1]$. Notice that if $c=0=b$, then $a=1$ and $T\left(\left(x_{1}, x_{2}\right),\left(y_{1}, y_{2}\right)\right)=x_{1} y_{1}$. Hence,

$$
\operatorname{Norm}(T)=\{( \pm(1, t), \pm(1, s)):-1 \leq t, s \leq 1\} .
$$

Let $c=0$ and $b>0$. Suppose that $((1, t),(1, y)) \in \operatorname{Norm}(T)$ for some $t, y \in[-1,1]$. It follows that

$$
1=|T((1, t),(1, y))| \leq|a+b t y| \leq a+b|t||y| \leq a+b=1,
$$

which implies that $1=t y$. Hence, $((1,1),(1,1)),((1,-1),(1,-1)) \in \operatorname{Norm}(T)$.

Suppose that $((1, t),(y, 1)) \in \operatorname{Norm}(T)$ for some $t, y \in[-1,1]$. It follows that

$$
1=|T((1, t),(y, 1))| \leq|a y+b t| \leq a|y|+b|t| \leq a+b=1
$$

which implies that $t=y= \pm 1$. Hence, $((1,1),(1,1)),((1,-1),(-1,1)) \in \operatorname{Norm}(T)$.

Suppose that $((t, 1),(y, 1)) \in \operatorname{Norm}(T)$ for some $t, y \in[-1,1]$. It follows that

$$
1=|T((t, 1),(y, 1))| \leq|a t y+b| \leq a|t||y|+b \leq a+b=1,
$$

which implies that $1=t y$. Hence, $((1,1),(1,1)),((-1,1),(-1,1)) \in \operatorname{Norm}(T)$. By Lemma 2,

$$
\operatorname{Norm}(T)=\{( \pm(1,1), \pm(1,1)),( \pm(1,-1), \pm(1,-1))\}
$$


It follows that

$$
1=|T((1, t),(1, y))| \leq|a+b t y+c(t+y)| \leq a+b|t||y|+c(|t|+|y|) \leq a+b+2 c=1,
$$

which implies that $1=t y=t=y$. Hence, $((1,1),(1,1)) \in \operatorname{Norm}(T)$.

Suppose that $((1, t),(y, 1)) \in \operatorname{Norm}(T)$ for some $t, y \in[-1,1]$. It follows that

$$
1=|T((1, t),(y, 1))| \leq|a y+b t+c(1+t y)| \leq a|y|+b|t|+c(1+|t||y|) \leq a+b+2 c=1,
$$

which implies that $1=t y=t=y$. Hence, $((1,1),(1,1)) \in \operatorname{Norm}(T)$.

Suppose that $((t, 1),(y, 1)) \in \operatorname{Norm}(T)$ for some $t, y \in[-1,1]$. It follows that

$$
1=|T((t, 1),(y, 1))| \leq|a t y+b+c(1+t y)| \leq a|t||y|+b+c(1+|t||y|) \leq a+b+2 c=1,
$$

which implies that $1=t y=t=y$. Hence, $((1,1),(1,1)) \in \operatorname{Norm}(T)$. By Lemma 2 ,

$$
\operatorname{Norm}(T)=\{( \pm(1,1), \pm(1,1))\}
$$

Case 2: $b<0$

Subcase 1: $1=|a+b|+2|c|>|a-b|$.

By Theorem A,

$$
\|T\|=1=|a+b|+2|c|=a+b+2 c>|a-b|=a-b .
$$

Notice that $c>|b|>0$ and $a \geq|b|>0$.

Let $c<\frac{1}{2}$. Suppose that $((1, t),(1, y)) \in \operatorname{Norm}(T)$ for some $t, y \in[-1,1]$. Then,

$$
1=|T((1, t),(1, y))|=|a+b t y+c(t+y)|=|y(c-|b| t)+a+c t| .
$$

Notice that $c-|b| t \neq 0$ because $c>|b|$. Suppose that $1=T((1, t),(1, y))$. Then

$$
y=\frac{1-a-c t}{c-|b| t}, 1=\frac{1-a-c}{c-|b|} \leq t .
$$

Hence, $t=1=y$ and $((1,1),(1,1)) \in \operatorname{Norm}(T)$. Suppose that $-1=T((1, t),(1, y))$. Then,

$$
y=\frac{-1-a-c t}{c-|b| t}, t \leq \frac{-1-a+c}{c+|b|}=-1 .
$$

Hence,

$$
t=-1, y=\frac{-1-a+c}{c+|b|}<-1
$$

a contradiction. Hence, there are no norming points of $T$ in this case.

Suppose that $((1, t),(y, 1)) \in \operatorname{Norm}(T)$ for some $t, y \in[-1,1]$. Then,

$$
1=|T((1, t),(y, 1))|=|a y+b t+c(1+t y)|=|y(a+c t)+c+b t| .
$$

We claim that $a+c t \neq 0$ for all $t \in[-1,1]$. Otherwise, there is $t \in[-1,1]$ such that $a+c t=0$. Then,

$$
\pm 1=c+b t=c+b\left(\frac{-a}{c}\right)=c+\frac{a|b|}{c} .
$$


Hence, $c=| \pm c|=c^{2}+a|b|=c^{2}+a(a+2 c-1)$, so

$$
0=c^{2}+(2 a-1) c+a(a-1)=(c+a)(c+a-1),
$$

which shows that $c=1-a$. It follows that $1=a+b+2 c=a+b+2(1-a)=2-a+b$, so $a-b=1$, a contradiction.

Suppose that $1=T((1, t),(y, 1))$. Then, $y=\frac{1-c-b t}{a+c t}$. If $a+c t>0$, then $1-c-b t \leq a+c t$, which implies that $t \geq \frac{1-a-c}{b+c}=1$. Hence, $t=1=y$. Hence, $((1,1),(1,1)) \in \operatorname{Norm}(T)$. Let $a+c t<0$. Then, $-1<t<0$ and $1-c-b t \leq-a-c t$. It follows that

$$
2 a+b+c=1+a-c \leq(b-c) t \leq|(b-c) t|<|b-c|=|b|+c,
$$

which implies that $a<|b|$, a contradiction. Hence, there are no norming points of $T$ in this case. Suppose that $-1=T((1, t),(y, 1))$. Then, $y=\frac{-1-c-b t}{a+c t}$. If $a+c t>0$, then $-a-c t \leq$ $-1-c-b t$, which implies that $t \geq \frac{1-a+c}{-b+c}>1$, a contradiction. Hence, there are no norming points of $T$ in this case. Let $a+c t<0$. Then, $-1<t<0$ and $-1-c-b t \geq a+c t$. It follows that $-(b+c)=-|b+c|<(b+c) t<-1-a-c$, which implies that $1<b-a<0$, a contradiction. Hence, there are no norming points of $T$ in this case.

Suppose that $((t, 1),(y, 1)) \in \operatorname{Norm}(T)$ for some $t, y \in[-1,1]$. Then,

$$
1=|T((t, 1),(y, 1))|=|a t y+b+c(t+y)|=|y(c+a t)+b+c t| .
$$

We claim that $c+a t \neq 0$ for all $t \in[-1,1]$. Otherwise, there is $t \in[-1,1]$ such that $c+a t=0$. Then,

$$
1 \geq|t|=\frac{c}{a}, \pm 1=b+c t=b+c\left(\frac{-c}{a}\right),
$$

so $a^{2} \geq c^{2}=a(b \pm 1)$, which implies that $a \geq \max \{b+1, b-1\}=b+1$, a contradiction since $a-b<1$. Suppose that $1=T((t, 1),(y, 1))$. Then, $y=\frac{1-b-c t}{c+a t}$. If $c+a t>0$, then $1-b-c t \leq$ $c+a t$, which implies that $t \geq \frac{1-b-c}{a+c}=1$. Hence, $t=1=y$ and $((1,1),(1,1)) \in \operatorname{Norm}(T)$. If $c+a t<0$, then $c+a t \leq 1-b-c t$, which implies that $t \leq \frac{1-b-c}{a+c}=1$. Thus, $t=1$ and $0<c+a=c+a t<0$, a contradiction. Hence, there are no norming points of $T$ in this case.

Suppose that $-1=T((t, 1),(y, 1))$. Then, $y=\frac{-1-b-c t}{c+a t}$. If $c+a t>0$, then $-c-a t \leq-1-$ $b-c t$, which implies that $(c-a) t \leq-1-b+c$. It follows that $-|c-a| \leq(c-a) t \leq-1-b+c$, so $a \leq|b|, c=\frac{1}{2}$, a contradiction. Hence, there are no norming points of $T$ in this case. If $c+a t<0$, then $c+a t \leq-1-b-c t$, which implies that $t \leq \frac{-1-b-c}{a+c}<-1$, a contradiction. Thus, there are no norming points of $T$ in this case. Therefore, Norm $(T)=\{( \pm(1,1), \pm(1,1)\}$. Let $c=\frac{1}{2}$. Then,

$$
T\left(\left(x_{1}, x_{2}\right),\left(y_{1}, y_{2}\right)\right)=a x_{1} y_{1}-a x_{2} y_{2}+\frac{1}{2}\left(x_{1} y_{2}+x_{2} y_{1}\right)
$$

for some $0<a<\frac{1}{2}$. We will show that

$$
\operatorname{Norm}(T)=\{( \pm(1,1), \pm(1,1)),( \pm(1,-1), \pm(1,-1))\} .
$$

Suppose that $((1, t),(1, y)) \in \operatorname{Norm}(T)$ for some $t, y \in[-1,1]$. Then,

$$
1=|T((1, t),(1, y))|=\left|y\left(\frac{1}{2}-a t\right)+a+\frac{1}{2} t\right| .
$$

Hence,

$$
y=\frac{ \pm 1-a-\frac{1}{2} t}{\frac{1}{2}-a t} .
$$


Notice that if $1=T((1, t),(1, y))$, then $t=1=y$ and that if $-1=T((1, t),(1, y))$, then $t=-1=y$. Hence,

$$
((1,1),(1,1)),((1,-1),(1,-1)) \in \operatorname{Norm}(T) .
$$

Suppose that $((1, t),(y, 1)) \in \operatorname{Norm}(T)$ for some $t, y \in[-1,1]$. Then,

$$
1=|T((1, t),(y, 1))|=\left|y\left(a+\frac{1}{2} t\right)+\frac{1}{2}-a t\right| .
$$

Observe that $a+\frac{1}{2} t \neq 0$ for all $t \in[-1,1]$. Otherwise. $a+\frac{1}{2} t=0$ for some $t \in[-1,1]$. Then, $\pm 1=\frac{1}{2}+2 a^{2}<1$, a contradiction. Therefore,

$$
y=\frac{ \pm 1-\frac{1}{2}+a t}{a+\frac{1}{2} t} .
$$

Suppose that $1=T((1, t),(y, 1))$. If $a+\frac{1}{2} t>0$, then $t=1=y$ and that if $a+\frac{1}{2} t<0$, then $t=-1=y$. Hence,

$$
((1,1),(1,1)),((1,-1),(1,-1)) \in \operatorname{Norm}(T) .
$$

Notice that if $-1=T((1, t),(y, 1))$, there are no norming points of $T$.

Suppose that $((t, 1),(y, 1)) \in \operatorname{Norm}(T)$ for some $t, y \in[-1,1]$. Then,

$$
1=|T((t, 1),(y, 1))|=\left|y\left(\frac{1}{2}+a t\right)-a+\frac{1}{2} t\right| .
$$

Hence,

$$
y=\frac{ \pm 1+a-\frac{1}{2} t}{\frac{1}{2}+a t} .
$$

Notice that if $1=T((t, 1),(y, 1))$, then $t=1=y$ and that if $-1=T((t, 1),(y, 1))$, then $t=-1=y$. Thus,

$$
\operatorname{Norm}(T)=\{( \pm(1,1), \pm(1,1)),( \pm(1,-1), \pm(1,-1))\} .
$$

Subcase 2: $|a+b|+2|c|=|a-b|=1$.

Notice that $T\left(\left(x_{1}, x_{2}\right),\left(y_{1}, y_{2}\right)\right)=a x_{1} y_{1}-(1-a) x_{2} y_{2}+(1-a)\left(x_{1} y_{2}+x_{2} y_{1}\right)$ with $\frac{1}{2} \leq a<1$.

If $a=\frac{1}{2}$, then $T\left(\left(x_{1}, x_{2}\right),\left(y_{1}, y_{2}\right)\right)=\frac{1}{2} x_{1} y_{1}-\frac{1}{2} x_{2} y_{2}+\frac{1}{2}\left(x_{1} y_{2}+x_{2} y_{1}\right)$. Hence,

$$
\begin{aligned}
\operatorname{Norm}(T)= & \{( \pm(1,-1), \pm(s, 1)),( \pm(s, 1), \pm(1,-1)),( \pm(1, t), \pm(1,1)) \\
& ( \pm(1,1), \pm(1, t)):-1 \leq s, t \leq 1\} .
\end{aligned}
$$

Let $\frac{1}{2}<a$. Suppose that $((1, t),(1, y)) \in \operatorname{Norm}(T)$ for some $t, y \in[-1,1]$. Then,

$$
1=|T((1, t),(1, y))|=|a-(1-a) t y+(1-a)(t+y)|=|y(1-a)(1-t)+a+(1-a) t| .
$$

Therefore, $y=\frac{ \pm 1-a-(1-a) t}{(1-a)(1-t)}$. Suppose that $1=T((1, t),(1, y))$. Then, $y=1$ for all $t \in[-1,1]$. Hence, $((1, t),(1,1)) \in \operatorname{Norm}(T)$ for $-1 \leq t \leq 1$. Suppose that $-1=T((1, t),(1, y))$. Then,

$$
y=\frac{-1-a-(1-a) t}{(1-a)(1-t)}<-1,
$$

which is a contradiction. Thus, there are no norming points of $T$ in this case. 
Suppose that $((1, t),(y, 1)) \in \operatorname{Norm}(T)$ for some $t, y \in[-1,1]$. Then,

$$
1=|T((1, t),(y, 1))|=|y(a+(1-a) t)+(1-a)(1-t)| .
$$

Notice that $a+(1-a) t \neq 0$ for all $t \in[-1,1]$ because $a>\frac{1}{2}$. Hence, $y=\frac{ \pm 1-(1-a)(1-t)}{a+(1-a) t}$. Suppose that $1=T((1, t),(y, 1))$. Then, $y=1$ for all $x \in[-1,1]$. Thus, $((1, t),(1,1)) \in \operatorname{Norm}(T)$ for $-1 \leq t \leq 1$.

Suppose that $-1=T((1, t),(y, 1))$. Then,

$$
y=1-\frac{2}{a+(1-a) t} \leq 1-\frac{2}{a+(1-a)|t|} \leq 1-\frac{2}{a+(1-a)}=-1 .
$$

Then, $y=-1, t=1$ Hence, $((1,1),(-1,1)) \in \operatorname{Norm}(T)$.

Suppose that $((t, 1),(y, 1)) \in \operatorname{Norm}(T)$ for some $t, y \in[-1,1]$. Then,

$$
1=|T((t, 1),(y, 1))|=|y(a t+(1-a))-(1-a)(1-t)| .
$$

We claim that that $a t+(1-a) \neq 0$ for all $t \in[-1,1]$. Otherwise, there is $t \in[-1,1]$ such that $a t+(1-a)=0$. Then,

$$
t=\frac{-(1-a)}{a}, \pm 1+(1-a)\left(1+\frac{1-a}{a}\right)=0,
$$

which is impossible. Hence,

$$
y=\frac{ \pm 1+(1-a)(1-t)}{(1-a)+a t} .
$$

Suppose that $1=T((t, 1),(y, 1))$. Then,

$$
y=\frac{1+(1-a)(1-t)}{a t+(1-a)} .
$$

If $a t+(1-a)>0$, then $1+(1-a)(1-t) \leq a t+(1-a)$. Hence, $t=1=y$. Therefore, $((1,1),(1,1)) \in \operatorname{Norm}(T)$. If $a t+(1-a)<0$, then $-a t-(1-a) \geq 1+(1-a)(1-t)$. Hence, $t \leq \frac{3-2 a}{1-2 a}<-1$ a contradiction. Thus, there are no norming points of $T$ in this case.

Suppose that $-1=T((t, 1),(y, 1))$. Then,

$$
y=\frac{a t-(a+t)}{a t-(a-1)} \geq 1,
$$

so $y=1, t=-1$. Hence, $((-1,1),(1,1)) \in \operatorname{Norm}(T)$. Therefore,

$$
\begin{aligned}
\operatorname{Norm}(T)= & \{( \pm(1, t), \pm(1,1)),( \pm(1,1), \pm(1, t)),( \pm(1,1), \pm(1,-1)) \\
& ( \pm(1,-1), \pm(1,1)):-1 \leq t \leq 1\}
\end{aligned}
$$

Subcase 3: $|a+b|+2|c|<|a-b|=1$.

By Theorem A,

$$
|a+b|+2|c|=a-|b|+2 c<|a-b|=a+|b|=1=\|T\| .
$$

Notice that $a \geq|b|>c$. Suppose that $((1, t),(1, y)) \in \operatorname{Norm}(T)$ for some $t, y \in[-1,1]$. Then,

$$
1=|T((1, t),(1, y))|=|a+b t y+c(t+y)|=|y(c-|b| t)+a+c t| .
$$


We claim that $c-|b| t \neq 0$. Otherwise, there is $t \in[-1,1]$ such that $c-|b| t=0$. Then, $t=\frac{c}{|b|}, \pm 1-a-c t=0$. Hence,

$$
0=\left( \pm 1-a-c\left(\frac{c}{|b|}\right)\right)|b|
$$

so $0=|b|^{2}-c^{2}$ or $-(1+a)|b|=c^{2}$, which imply that $|b|=c$ or $c^{2}<0$. These are contradictions.

Suppose that $1=T((1, t),(1, y))=a+b t y+c(t+y)$. If $c-|b| t>0$, then

$$
y=\frac{1-a-c t}{c-|b| t}, t \leq \min \left\{\frac{1-a-c}{c-|b|}, \frac{1-a+c}{c+|b|}\right\}=\min \{-1,1\}=-1 .
$$

Hence, $t=-1, y=1$, and $((1,-1),(1,1)) \in \operatorname{Norm}(T)$. If $c-|b| t<0$, then

$$
y=\frac{1-a-c t}{c-|b| t}, 1=\frac{1-a+c}{c+|b|} \leq t \leq \frac{1-a-c}{c-|b|}=-1
$$

a contradiction. Hence, there are no norming points of $T$ in this case.

Suppose that $-1=T((1, t),(1, y))$. If $c-|b| t>0$, then

$$
y=\frac{-1-a-c t}{c-|b| t}, t \leq \min \left\{\frac{-1-a-c}{c-|b|}, \frac{-1-a+c}{c+|b|}\right\}=\frac{-1-a+c}{c+|b|}<-1,
$$

a contradiction. Hence, there are no norming points of $T$ in this case. If $c-|b| t<0$, then $y=\frac{-1-a-c t}{c-|b| t}>1$ because $(|b|-c) t \leq|b|-c \leq|b|<1+a+c$. This is a contradiction. Therefore, there are no norming points of $T$ in this case.

Suppose that $((1, t),(y, 1)) \in \operatorname{Norm}(T)$ for some $t, y \in[-1,1]$. Then,

$$
1=|T((1, t),(y, 1))|=|a y+b t+c(1+t y)|=|y(a+c t)+c+b t| .
$$

Since $a>c, a+c t \neq 0$. Suppose that $1=T((1, t),(y, 1))$. Then,

$$
y=\frac{1-c-b t}{a+c t}, t \leq \frac{1-a-c}{c-|b|}=-1 .
$$

Hence, $t=-1, y=1$, and $((1,-1), \pm(1,1)),(-(1,1), \pm(1,-1)) \in \operatorname{Norm}(T)$.

Suppose that $-1=T((1, t),(y, 1))$. Then,

$$
y=\frac{-1-c-b t}{a+c t}, 1=\frac{-1-a+c}{c+|b|} \leq t .
$$

Therefore, $t=1, y=-1$, and $((1,1),(-1,1)) \in \operatorname{Norm}(T)$. We claim that $c+a t \neq 0$ for all $t \in[-1,1]$. Indeed, assume that $c+a t=0$ for some $t \in[-1,1]$. Then, $\pm 1=b+c t=b-c\left(\frac{c}{a}\right)$, which implies that $\pm a=a b-c^{2}$. Hence, $a=| \pm a|=\left|a b-c^{2}\right|=a|b|+c^{2}$, so $a^{2}=a(1-|b|)=c^{2}$, so $a=c$, which is impossible since $a>c$. Suppose that $((t, 1),(y, 1)) \in \operatorname{Norm}(T)$ for some $t, y \in[-1,1]$. Then,

$$
1=|T((t, 1),(y, 1))|=|a t y+b+c(t+y)|=|y(c+a t)+b+c t| .
$$


Suppose that $1=T((t, 1),(y, 1))=a t y+b+c(t+y)$. Let $c>0$. Then, $y=\frac{1-b-c t}{c+a t}$. Note that if $c+a t>0$, then $t \geq \frac{1-b-c}{a+c}>1$. Hence, there are no norming points of $T$ in this case. Let $c+a t<0$. Then, $t \leq \frac{1-b+c}{c-a}<-1$. Thus, there are no norming points of $T$ in this case.

Suppose that $-1=T((t, 1),(y, 1))$. Then, $y=\frac{-1-b-c t}{c+a t}$. If $c+a t>0$, then

$$
t \geq \max \left\{\frac{1+b-c}{a-c}, \frac{-1-b-c}{a+c}\right\}=\max \{1,-1\}=1 .
$$

Hence, $t=1, y=-1$, and $((1,1),(-1,1)) \in \operatorname{Norm}(T)$. If $c+a t<0$, then

$$
t \leq \min \left\{\frac{1+b-c}{a-c}, \frac{-1-b-c}{a+c}\right\}=\min \{1,-1\}=-1 \text {. }
$$

Therefore, $t=-1, y=1$, and $((-1,1),(1,1)) \in \operatorname{Norm}(T)$. By Lemma 2,

$$
\operatorname{Norm}(T)=\{( \pm(1,-1), \pm(1,1)),( \pm(1,1), \pm(1,-1))\}
$$

\section{REFERENCES}

1. R.M. Aron, C. Finet, E. Werner, Some remarks on norm-attaining n-linear forms, Function spaces (Edwardsville, IL, 1994), 19-28, Lecture Notes in Pure and Appl. Math., V. 172, Dekker, New York, 1995.

2. E. Bishop, R. Phelps, A proof that every Banach space is subreflexive, Bull. Amer. Math. Soc., 67 (1961), 97-98.

3. Y.S. Choi, S.G. Kim, Norm or numerical radius attaining multilinear mappings and polynomials, J. London Math. Soc., 54 (1996), №2, 135-147.

4. Y.S. Choi, S.G. Kim, The unit ball of $\mathcal{P}\left({ }^{2} l_{2}^{2}\right)$, Arch. Math., Basel, 71 (1998), 472-480.

5. S. Dineen, Complex analysis on infinite dimensional spaces, Springer-Verlag, London, 1999.

6. M. Jimenez Sevilla, R. Paya, Norm attaining multilinear forms and polynomials on preduals of Lorentz sequence spaces, Studia Math., 127 (1998), 99-112.

7. S.G. Kim, The unit ball of $\mathcal{L}_{s}\left({ }^{2} l_{\infty}^{2}\right)$, Extracta Math., 24 (2009), 17-29.

8. S.G. Kim, The norming set of a polynomial in $\mathcal{P}\left({ }^{2} l_{\infty}^{2}\right)$, Honam Math. J., 42 (2020), №3, 569-576.

Department of Mathematics, Kyungpook National University, Daegu 702-701, Republic of Korea sgk317@knu.ac.kr 